\title{
Volleyball Action Extraction and Dynamic Recognition Based on Gait Tactile Sensor
}

\author{
Limin Qi \\ College of Sports Science, Harbin Normal University, Harbin, 150025 Heilongjiang, China \\ Correspondence should be addressed to Limin Qi; 474949287@hrbnu.edu.cn
}

Received 13 April 2021; Revised 27 April 2021; Accepted 15 May 2021; Published 2 June 2021

Academic Editor: Mu Zhou

Copyright (c) 2021 Limin Qi. This is an open access article distributed under the Creative Commons Attribution License, which permits unrestricted use, distribution, and reproduction in any medium, provided the original work is properly cited.

\begin{abstract}
At present, the industry research of volleyball technology is relatively in-depth, and the analysis of the muscle strength characteristics and coordination of the jumping ball is less, which is not conducive to the control of technical movements. This study used a wireless portable surface EMG tester (16 lines) to analyze the EMG of the main muscle groups in athletes' volleyball and conducted a video synchronization test method to find the position of the human body. Therefore, a backgroundbased frame difference method is proposed to detect the position and obtain the precise position of the human body. Experiments show that the background-based three-frame difference method effectively eliminates the "hole" effect of the original three-frame difference method and provides an accurate and complete framework for identifying the human body. Adjust the recognition frame according to the proportion of the human body in the image, and use the predefined parameters of the severe frame to perform forward/volleyball background segmentation. The novelty of this document lies in the completion of the complete human body placement of the above three tasks, precapture/background segmentation, and an improved human body position estimation algorithm to extract the human body pose from the video. First, locate the human body in each frame of the video, and then, perform the process of estimating the position of the graphic model based on the color and texture of the unit. After recognizing the gesture of each image in the video, the recognition result will be displayed. Experiments show that after detecting the position of the human body, the predefined frame setting process of the tomb is carried out in two steps, which improves the automation of the human body image detection algorithm, effectively extracts the human motion video, and increases the motion capture rate by more than $30 \%$, to provide a useful reference for the improvement of college volleyball players' movement skills and training competitions.
\end{abstract}

\section{Introduction}

The volleyball game began in 1895, and its founder was Holly Walker of Massachusetts, USA, the secretary of the YMCA, William Morgan. At the beginning, his idea was to choose a moderate, moderate sport. This is a leisure sport that can meet the needs of people who cannot adapt to the intense basketball game. Casual timetable, but as time goes by, volleyball has gained more and more public recognition and is developing rapidly. After continuous reform and improvement, it has become a world-class sport. Keeping pace with the times and looking forward to the future, volleyball is also developing rapidly. In addition to the conventional six-sided volleyball and beach volleyball, various volleyball sports are widely used, such as soft volleyball and air volleyball. Volleyball has a variety of forms and comprehensive skills. In China, since the Chinese women's volleyball team won a difficult championship in the 2004 Athens Olympics, China has always had an unlimited volleyball boom. The national sand queuing performance has also improved by leaps and bounds, and soft volleyball has become a competition event in various middle schools. It has gradually appeared in various city offices and enterprises and has become an official event for sports workers. 
For humans, understanding the posture of volleyball can be easily solved, but for computers, this understanding is indeed quite difficult. So far, there is no algorithm or system that can accurately describe the motion of an image with a close human comprehension ability under few restrictions. Therefore, this article attempts to do some work in this direction and achieve some results.

Ning believes that after more than 20 years of development, my country's volleyball league has continuously adapted to the reform of the International Volleyball Federation, and social attention and respect have greatly increased. Professional sports companies have intervened, including the Hawkeye referee assistance system, the introduction of foreign aid, and the reform of the competition system. These measures are bound to accelerate the professionalization of my country's volleyball championships, activate the volleyball market, and promote the recurrence of volleyball in our country, but the human body research of volleyball is not perfect [1]. Liu proposed an ultrawideband position calibration method based on motion detection. The realization of this method is based on two experiments. One is to use a total station to check the accuracy of the motion detection system, and through Bursa seven-coordinate parameter conversion, the model will process experimental data, which shows that the accuracy of the motion detection system meets the requirements and can be used to calibrate the motion detection system. The accuracy of the UWB indoor installation system: the second is to calibrate the accuracy of the UWB indoor positioning system through the motion detection system detected by the motion detection system. The data is real. Compare the data recorded by the UWB indoor installation system with the data recorded by the motion detection system. However, the accuracy of the experiment is not high and needs to be improved [2,3]. Wang proposed a new MOCAP algorithm to recover data distortion. The algorithm first preprocesses the MOCAP data so that the converted data represents the change in the relative position of adjacent markers to receive the volleyball length limiter and then uses it. The sparse display and the limitation of the length of volleyball are used for dictionary training, and finally, the trained dictionary is used to recover the lost data. But this method requires a lot of time and cannot get results quickly [4].

The innovation of this article is to use eye tracking technology to explore and analyze the cognitive processing characteristics of volleyball players of different sports levels when judging the point of serve. It provides a theoretical basis for the cognitive processing characteristics of volleyball players and can be used as a reference for the selection of athletes. The expected research hypothesizes that there are differences in cognitive processing characteristics of volleyball players when judging the drop points of different serve offenses. Therefore, human motion detection and volleyball movement changes can be studied as independent topics or as a comprehensive study of the various steps of a topic. The experimental results of this method show that the target step rate is increased by more than $60 \%$. This article is mainly devoted to the study of target detection and extraction in the analysis of moving objects.

\section{Content and Method of Gait Tactile Sensor}

\subsection{Main Content of the Gait Tactile Sensor System}

(1) To systematically develop and research gait tactile sensors and discuss the relevant background and research significance of the topic [5]

(2) Based on the previous work, a simple large-area robot sensor array was developed, focusing on the development of detection circuits, signal processing circuits, and signal receiving circuits for receiving and processing signals

(3) The complete virtual instrument technology data acquisition and analysis system based on NI PCI6259 data acquisition card can provide data acquisition, analysis and processing, real-time control, and synchronization control. Use OpenGL technology to design the screen software system [6]

(4) The integrity, advantages, and disadvantages of the gait tactile sensor and hardware and software systems are tested through experiments

(5) Analyze the error of the experimental results, discuss the source of the error, summarize the remaining problems, and propose the direction of the next step [7]

2.2. Literature Data Method. Retrieval and screening of relevant literature data are based on "volleyball physical training," "jump serve technique," "core strength," and keywords through Chinese journal databases such as CNKI and Wanfang and the library of Shandong Institute of Physical Education [8,9]; nearly 110 related documents on the use of core strength in volleyball were obtained, sorted out, refined, and summarized, through the sorting and analysis of reference materials, mastering the research and analysis of core strength and technical movements in volleyball, focusing on the analysis of the concept of core strength and the theoretical basis of training such as jump serve movement analysis, training methods, and stability factors $[10,11]$.

2.3. Expert Interview Method. On the basis of consulting relevant materials, in order to obtain sufficient theoretical basis such as scientific and reasonable measurement indicators and research methods, the purpose is to consult

(1) about the main factors affecting jump serve training [12]

(2) select measurement indicators related to volleyball jump serve training

(3) issues related to the selection of core strength training methods

This was done through visits to volleyball coaches and related academic experts, according to the starting point and feasibility, actual conditions, rationality and testing methods of this thesis research, the selection of evaluation 
indicators and training methods, and consulting experts' opinions $[13,14]$.

2.4. Questionnaire Survey Method. Before the experiment, in order to obtain reasonable experimental training methods and ensure the effectiveness of various indicators, a list of the core strength training for male volleyball players' jump serve training methods index selection order table was developed. The title of the topic is the research screening table of the influence of core strength training on the jump serve ability of male volleyball players, and the experimental training indicators are determined by the selection and evaluation of experts in all aspects of the volleyball field $[15,16]$. The design purpose of the questionnaire is to obtain effective index help and guiding suggestions that are conducive to improving the training methods of volleyball players' jump serve ability [17].

\section{Correlation Experiment of Gait Tactile Sensor}

In order to perceive the external environment quickly and accurately, many experts and scholars have developed various sensors according to different needs. The sensing principles can be divided into seven types, namely, piezoresistive effect, piezoelectric effect and pyroelectric effect, capacitive, magnetoelectric sensing, photoelectric sensing, mechanical sensing, and ultrasonic sensing. Sensitive materials are used for the conversion and sensitive components in the sensor design. Its performance directly affects the performance indicators of the sensor. Therefore, in order to be able to design a sensor that meets actual requirements, the first problem to be solved is to select the sensitive material $[18,19]$. In order to enable the flexible tactile sensor to have efficient sensing and energy conversion capabilities, this paper selects a piece of elastic silica gel as the sensitive element of the flexible tactile sensor and selects a strain gauge as the conversion element of the flexible tactile sensor. According to the requirements of the subject, various factors in the laboratory are comprehensively considered. Due to the advantages of simple structure, strong antifatigue ability, easy signal processing, and low signal hysteresis [20], the strain sensor is selected in this paper, as shown in Figure 1.

It is the schematic diagram of the initial tactile information collection circuit, where the dashed frame represents the unit volume of conductive rubber $K$ (its piezoresistive characteristic curve has been determined), $Q$ is the reference resistance [21,22], and $e$ is the regulated DC power supply. Since the voltage of $e$ and the resistance of $Q$ are constant, when we apply pressure to the conductive rubber $Q$, its resistance also changes so that the voltage $V$ shared on $Q$ changes.

$$
Q=Q_{C} \times K \cos e+V .
$$

In the first state, the sensitive unit is not under pressure, and the unit volume of conductive rubber $Q$ appears as an open switch in the circuit; in the second state, the sensitive unit is under pressure $Q 1$, and the voltage $V 1$ is read at $R$ at this time; in the third state, the sensitive unit is under pressure $Q 2$, read the voltage $V 2$ at $R$ at this time $[23,24]$.

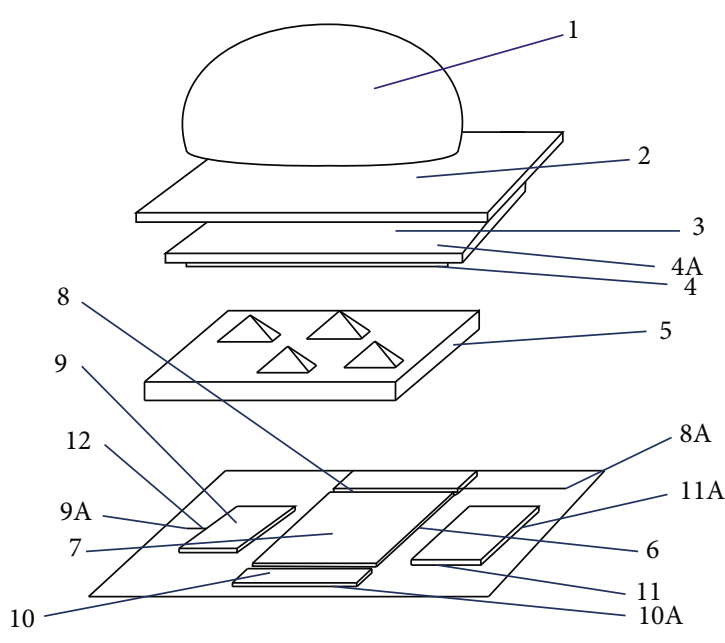

Figure 1: Gait tactile sensor.

$$
Q_{C}=Q 1+Q 2=\gamma \times Q 2-\gamma \times Q 1 .
$$

The value of the resistance $Q$ per unit volume of conductive rubber under pressure can be calculated from the formula, and the coefficient can be found from the piezoresistance curve according to this value.

When the resistance wire is under the action of the tensile force $F$, the resistance wire elongates $L$, the cross-sectional area decreases $C$, and the resistivity changes due to the change of the material lattice, which causes the resistance to also change $K$. Differentiate and get the relative change of resistance as [25]

$$
\frac{d Q}{Q}=\frac{d C}{C}=\frac{d F}{F}+Q^{C} .
$$

Among them, $L$ is the amount of change in length, expressed as strain $M$ as

$$
M=\frac{d L}{L}-\sqrt{M}+C .
$$

According to the mechanics of materials, in the elastic range, when the metal wire is under tension, the metal wire elongates in the axial direction and shortens in the radial direction so that $M$ is the axial strain of the metal resistance wire and $C$ is the radial direction of the metal resistance wire. Strain, so the relationship between axial strain and radial strain can be expressed as [26]

$$
\frac{d Q}{Q}=-\mu \frac{d L}{L}+\sqrt{M}=-\mu M .
$$

$\mu$ in the formula is Poisson's ratio of the metal resistance wire material, and the negative sign indicates that the strain direction is opposite. Substituting formula (3) into formula (5), we can get

$$
\frac{d R}{R}=(1+2 \mu) C+\frac{d F}{F}-\mu,
$$


where $R$ is the leakage resistance of the $K$ th row and $R$ is the leakage resistance of the $Z$ th column, expressed as

$$
\frac{1}{R_{i}}=\sum_{1}^{n} \frac{1}{r_{z}}+\frac{1}{R}
$$

In formulas (6) and (7), the insulation resistance is between $P$ and $U$ row and column electrodes. The analysis of the circuit shows that the circuit is a typical reverse addition circuit [27]:

$$
V_{\text {cout }}=-P_{1}\left(\frac{V}{R}+\frac{U}{R}\right)=-\frac{1}{R_{i}} \times V_{i} \text {. }
$$

The line scan method scans one line at a time, thereby greatly improving the readout speed of all information in a single tactile array $[28,29]$, laying the hardware foundation for the real-time performance of the entire system and improving the sampling speed while better solving the problem between rows and columns, the problem of crosstalk noise. Therefore, in this design, the line scan method is used to scan and collect the tactile signals. The circuit is mainly controlled by a set of pulse sequences, and the gating of eight analog switches is controlled by a six-bit counter [30-32].

\section{Volleyball Action Extraction and Dynamic Recognition Analysis}

An independent sample $T$ test was performed on the physical fitness indicators of the experimental group and the control group before the experiment. The test results showed that there was no significant difference in the physical fitness indicators of the two groups of athletes $(P>0.05)$, so the experimental group can be defined statistically. There is no significant difference in the physical fitness index of the athletes in the control group before the experiment.

From the results in Table 1, it can be seen that the training methods of the experimental group and the control group have certain differences and many connections in many comparison items. Not all traditional training methods are useless. It shows that there is no significant comparison between the two groups of feet in the vertical jump, approach height, and 30-meter sprint, indicating that the physical fitness indicators are the same.

From Figure 2, we can see that for the training of the core strength and serve speed of volleyball jump serve technology, different forms of training are used to improve the stability of the core muscle group of jump serve hits, so as to improve the stability of the athletes' jump serve hits sexual ability, thereby improving the speed and success rate of jump serve hits in training and competition.

It can be seen from Figure 3 that the use of traditional training methods has certain advantages and foundations, which are mainly to improve the maximum muscle strength by overcoming its own gravity and the external resistance of load bearing. The advantages of the training methods adopted in this article are mainly the action function in the field of training; the action mode has a variety of actions
TABLE 1: The statistical results of athletes' physical fitness indicators before the experiment.

\begin{tabular}{lccccc}
\hline Index & $\begin{array}{c}\text { Prone back } \\
\text { extension }\end{array}$ & $\begin{array}{c}\text { Sit- } \\
\text { ups }\end{array}$ & $\begin{array}{c}\text { Foot } \\
\text { jump }\end{array}$ & $\begin{array}{c}\text { Run } \\
\text { up }\end{array}$ & $\begin{array}{c}300 \mathrm{~m} \\
\text { sprint }\end{array}$ \\
\hline $\begin{array}{l}\text { Control } \\
\text { group }\end{array}$ & 37.95 & 47.24 & 52.36 & 35.26 & 40.12 \\
$\begin{array}{l}\text { Test group } \\
P \text { value }\end{array}$ & 32.77 & 46.45 & 39.33 & 30.16 & 51.23 \\
\hline
\end{tabular}

and the method is flexible; the muscle movement has the joint and coordinated force of the large and small muscle groups; the emphasis is on the power chain, coordination chain, joint flexibility and stability, action mode, and training purpose to improve the muscular endurance of the athletic ability and the stability of the specific technical action. The shortcomings of traditional training methods are the movement is relatively single and fixed, the single-joint muscle training, the emphasis is on absolute strength, separation from specific technical movements, and the training purpose is to train with intensity and improve physical fitness.

As shown in Table 2, in the results of the analysis of variance (ANOVA) on the three parameters of gait, the $P$ value is less than 0.05 , indicating that the three parameters have significant statistical differences in the three selfselected speed modes. The mean value and error distribution are shown in the figure. The results in the figure also show that the three parameters are statistically different in the three optional speed modes. Therefore, in the subsequent reliability analysis, the reliability of the three speed modes must be investigated separately.

As shown in Figure 4, the reliability coefficients from 2 to 10 measurements are calculated in each speed mode, and their values and changing trends are shown in Figure 2.3. The data in Table 2.3 is the statistical result of the number of measurements required to reach the ICC value of 0.75 and 0.9 in Figure 2.3. Obviously, in order to meet the ICC value of 0.75 , only 2 measurements are required in slow walking, normal walking, and fast walking modes; and in order to meet the ICC value of 0.9 , at least 3 measurements are required in slow walking mode; and in normal walking and fast walking modes, at least 2 measurements are needed.

The comparison between the gait parameters obtained from the research in Figure 5 and the existing research results is shown. From the comparison, it can be seen that the step length and pace are smaller than the previous research results, but this is consistent with the research results of Chiu, who also used Asian races as the experimental subjects. He limited speeds of 3,4 , and $5 \mathrm{~km} / \mathrm{h}$, respectively. It is slow walking, normal walking, and fast walking (Chiu and Wang, 2007). Obviously, the results of different races will be different (Braun et al., 1980). When two points on the touch sensor array are pressed, the waveform window displays two highlevel signals. The digital signal represents the corresponding row. The experimental data can also be seen from the saved text table, but due to the large amount of data in the table, it is inconvenient to display, so intuitive graphic display is adopted to prove the correctness of the experimental data. 


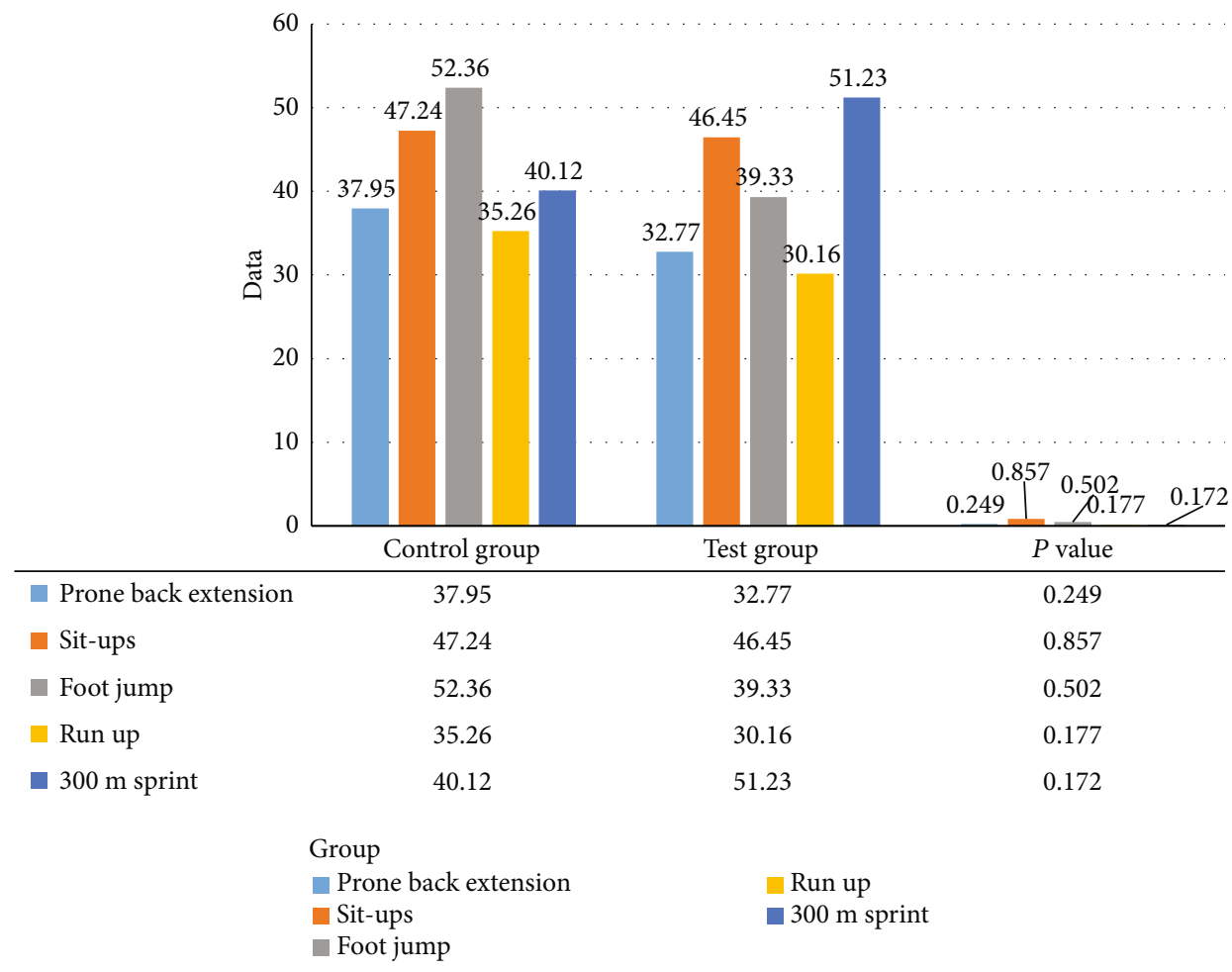

FIGURE 2: The statistical results of athletes' physical fitness indicators before the experiment.

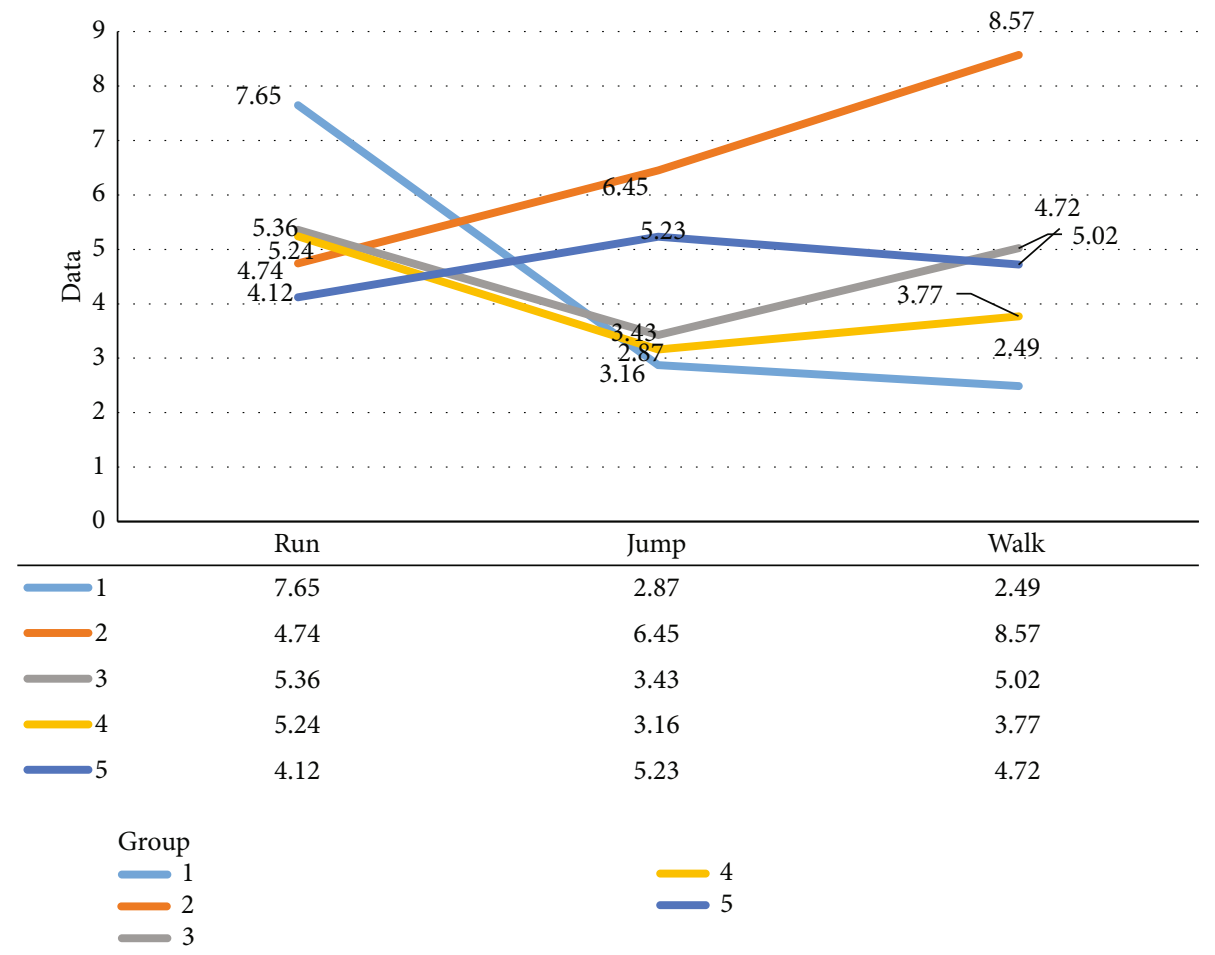

Figure 3: The statistical results of athletes' physical fitness indicators before the experiment. 
TABLE 2: The statistical results of athletes' physical fitness indicators before the experiment.

\begin{tabular}{lcccccc}
\hline & Slow & Normal & Fast & $\begin{array}{c}\text { Slow } \\
\text { normal }\end{array}$ & $\begin{array}{c}\text { Fast } \\
\text { normal }\end{array}$ & $\begin{array}{c}\text { Slow } \\
\text { fast }\end{array}$ \\
\hline $\begin{array}{l}\text { Step } \\
\text { length }\end{array}$ & 0.57 & 0.63 & 0.69 & 0.53 & 0.43 & 0.65 \\
Cadence & 0.21 & 0.13 & 0.31 & 0.25 & 0.21 & 0.35 \\
Velocity & 0.52 & 0.32 & 0.53 & 0.21 & 0.61 & 0.28 \\
\hline
\end{tabular}

In addition, we require the subject to be measured barefoot on the electronic trail, which may also be one of the reasons for the relatively small step length.

This experiment verifies that the electronic trail (also known as the digital track) has high reliability when collecting walking time and space parameters and will provide a good reference for researchers who use this instrument in the future. Different speed modes will affect the reliability of step length, step frequency, and pace. The slow walking, normal walking, and fast walking modes at the optional speed have high reliability. When the electronic trail is used in the experimental research of walking test, at least 3 measurements are required to meet the high reliability requirements under different speed modes.

There is relationship between vibration training under different vibration frequencies and the muscle strength of the upper limbs of the shoulder and elbow; there is relationship between vibration training and the explosive force and vibration frequency of the lower limbs of volleyball players. Then, through experimental training, we seek suitable vibration training strategies to maximize the athletes' whole body coordination, flexibility, bounce ability, and balance ability, in order to further apply suitable vibration strength training to the public level and improve the overall quality of citizens lay the foundation. Therefore, according to the research content, this thesis will determine the maximum strength of the shoulder and elbow joint, the flight time, the support time, the explosive force index of the lower limbs (lower extremity explosive force index = the flight time/support time), and other parameters for volleyball players and nonspecialized personnel under different vibration frequencies. The measurement results are compared horizontally and vertically to provide support for quantitative vibration training and volleyball players' strength research.

The intensity of myoelectric activity is triceps $>$ front deltoid $>$ biceps $>$ brachioradialis. The triceps of the upper limb muscles are very excited. The electric discharge is strong. Observing the technical structure of the action at this time, it can be found that the athlete's upper arm is swinging backwards forcefully. This swing makes the upper arm in a more favorable position for exerting force. It is for the next step to quickly swing forward and increase the support leg. The bottom force creates conditions. In the data acquisition system, to complete the signal measurement and analysis, you should first set the device name, sampling rate, trigger mode, and so on. In view of the more commonly used measurement requirements and signal types, we have designed the default values of the data acquisition parameters. Taking into account the diversification of user needs, users can also manually modify the parameter settings.

We can derive from Figure 6 that the jump-off buffer stage of the jump serve is the stage from the moment the jumping leg hits the ground to the maximum buffer moment of the knee joint of the jumping leg. Its main task is to actively complete the concessionary work of the muscles, store elastic potential energy, and serve for subsequent leg extensions. Make effective preparations. At this stage, the lower limbs have to withstand the greater impact of the ground, while the body maintains balance and the arms actively swing back to create the best working conditions for the subsequent kicking and stretching movements. Possible reasons for these results: the daily exercise load of the shoulder-elbow joint flexors of nonspecialized personnel is greater than that of the shoulder-elbow joint extensors, resulting in the training effect of the extensors being better than the flexors; the muscles are more suitable for low-frequency vibration training in the short term. Significant effects are seen inside. It can be seen that the effect of vibration training is still very significant, but it is not suitable for changing the vibration frequency in a short period of time. It takes a period of adaptation to achieve the optimal effect. These research results are all researches on the relationship between vibration training on shoulder and elbow joint upper limb muscle strength and vibration frequency, laying a foundation for further applying appropriate vibration strength training to the public level and improving the overall quality of citizens.

In the buffering stage, it mainly plays the role of pulling and braking the calf to buffer the horizontal speed. At the same time, braking the calf is more conducive to the formation of stable support for the ankle joint. The role of the gluteus maximus is relatively small in the lower limb muscles, with a contribution rate of only $6.18 \%$. The contribution rate of the EMG of the trunk muscles in the buffer phase is not high. The trapezius muscle, the external oblique muscle, and the erector spinae are all involved in the erection of the trunk and the extension of the spine. The sum of the three accounts for $16.41 \%$ of the total muscle activity tested. The myoelectric activity of the upper limb muscles is not high at this stage. The total myoelectric contribution rate accounts for $26.32 \%$ of all tested muscles, but the biceps myoelectric contribution rate accounts for $9.14 \%$, which produces stronger contraction and work. This is caused by the movement of the arm position during this process. These research results have laid the foundation for the research of vibration training on the relationship between the shoulder and elbow joint upper limb muscle strength and the vibration frequency and also laid the foundation for seeking suitable vibration training strategies to maximize the athlete's whole body coordination. Flexibility, jumping ability, balance, and other abilities lay the foundation for further applying appropriate vibration strength training to the public level and improving the overall quality of citizens.

Judging from the electromyographic characteristics of the jump serve of college volleyball players, the main muscle groups of the whole body are involved in the whole jump serve technique during the jump serve. Therefore, in the 


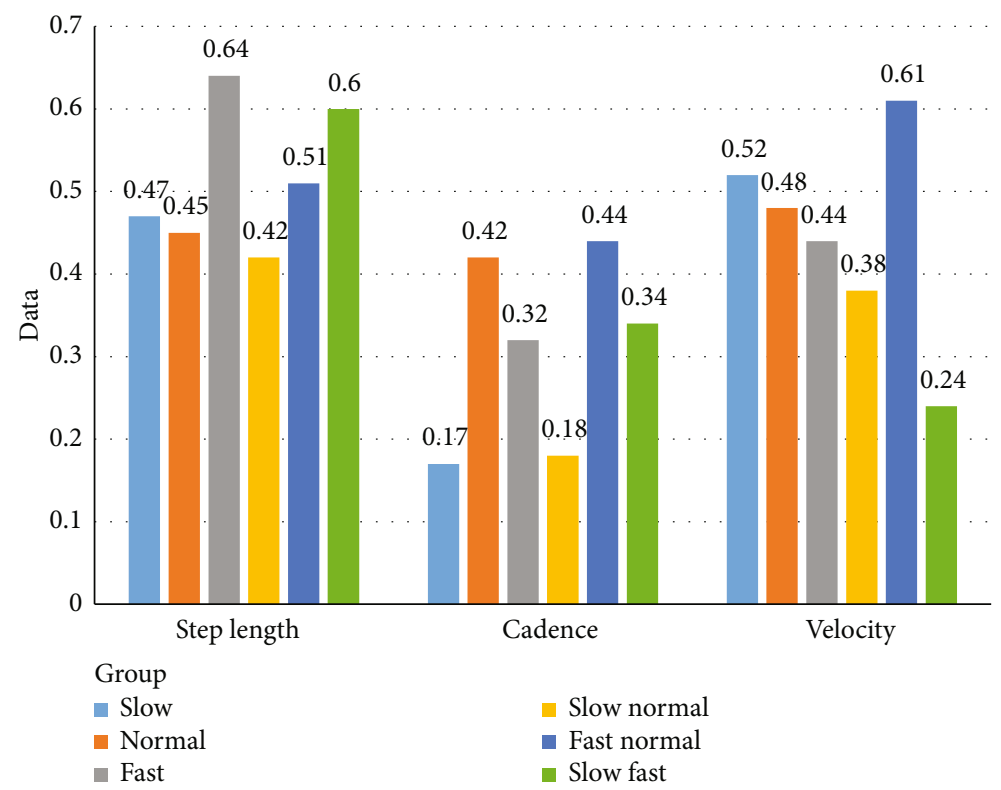

Figure 4: The statistical results of athletes' physical fitness indicators before the experiment.

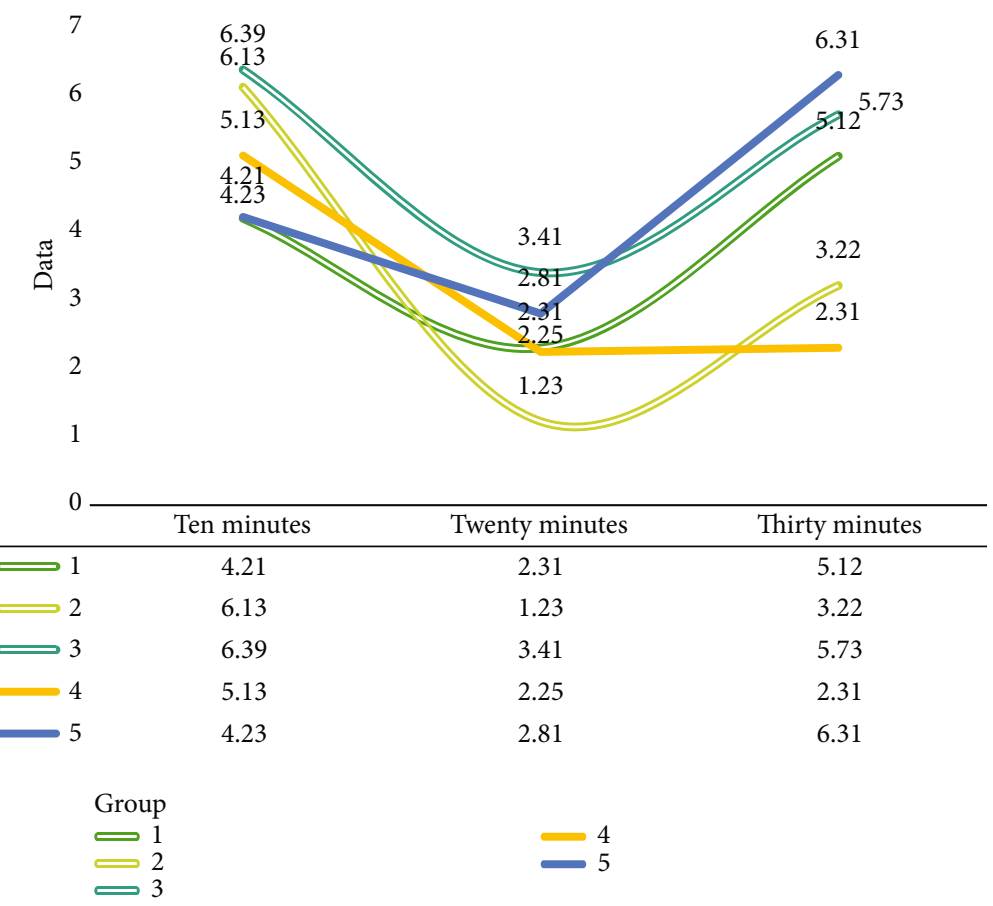

Figure 5: The statistical results of athletes' physical fitness indicators before the experiment.

process of muscle strength training of athletes, it is necessary to pay attention to all-round development. In their physical training, they should include depth jump training of different heights, weight-bearing training of different loads, and combined training formed by different training methods to achieve comprehensive development of the whole body. The role of muscle groups: the strength of the muscle group in the core area plays an important role in promoting the correct sequence of muscle exertion, reducing the loss of force transmitted between the shoulder and the arm, and ensuring the physical fitness required for the jump serve technique. Therefore, to strengthen the strength training in the core area, coaches should design and develop the core area muscle strength training methods on the basis of traditional strength 


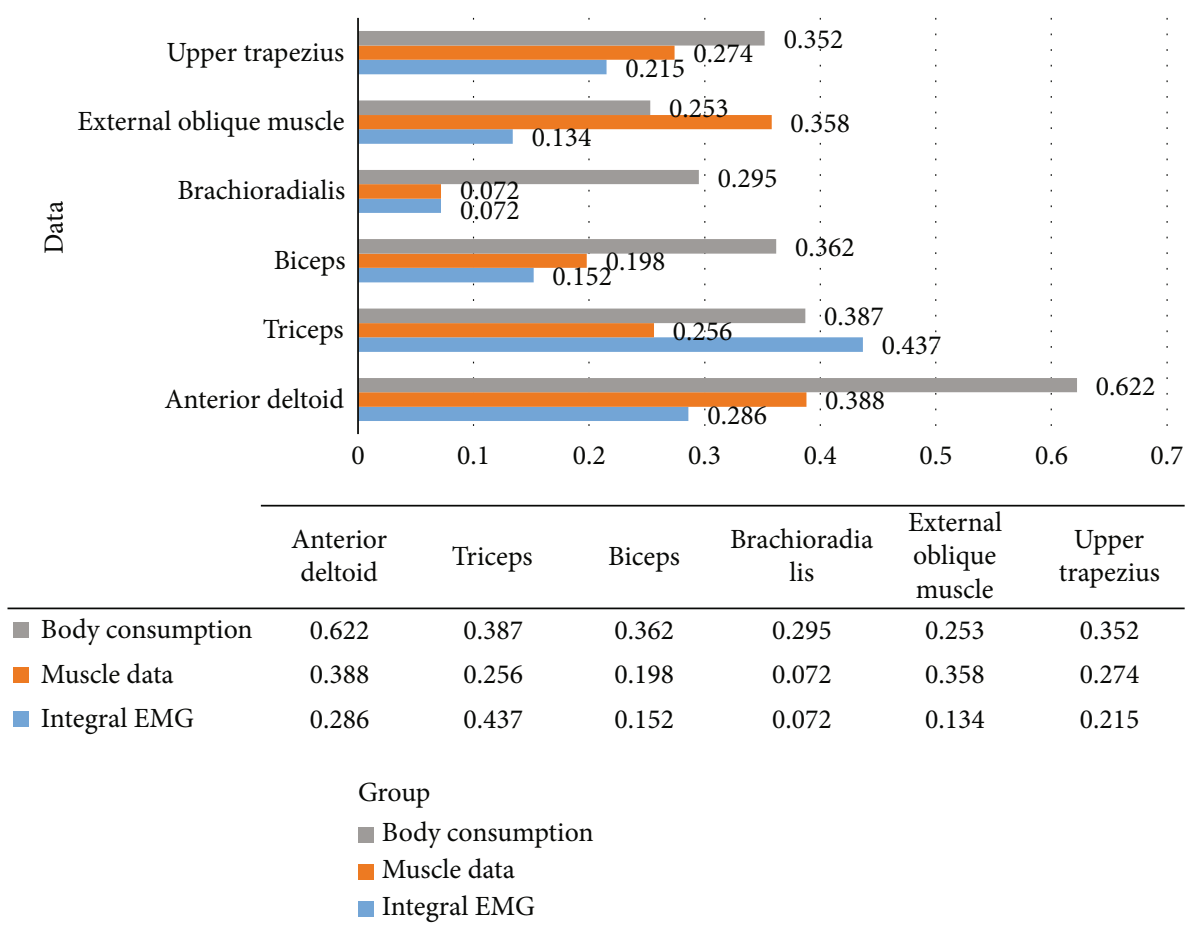

FIgURE 6: The integral EMG value of each muscle of the athlete in the last step of the jump serve and run-up.

training to supplement and improve the strength training system of volleyball players. From the perspective of the lower limb muscle force of college volleyball players, the coordinated development of lower limb joint muscle strength plays an important role in improving jumping efficiency. Although the special endurance training focuses on improving the flexibility of the lower limbs, the flexion endurance training of the lower limbs cannot be ignored. This is not only related to the coordination of lower extremity muscles to better improve muscle work efficiency and successfully complete jumps but also one of the important methods to prevent sports injuries.

According to the characteristics of the circuit and the degree of integration, MOS analog switch integrated circuits can be divided into many types. The types, names, and characteristics of commonly used analog switch integrated circuits are now listed in Table 3.

According to the needs of the experiment, we chose the CD4051 one-of-eight analog switch as the chip to control the switch circuit. Due to space limitations, only CD4051 is described in detail below. For details of CD4097, please refer to its technical data. CD4051 is a two-way analog switch. In the integrated circuit, there are 4 independent analog switches that can control the transmission of digital and analog signals. Each switch has an input port and an output port. They can be used instead. There is also a strobe terminal (also called a control terminal). When the selected communication terminal is high level, the switch is turned on. The selected communication terminal is usually in an energy-saving state, and the switch is turned off. Do not float the terminal strobe light when using it.
Touch sensors are one of the most important types of robot sensors, and their design also follows the basic principles of sensor design. It is mainly composed of sensitive components, conversion components, and signal conditioning and measurement circuits. The main components of the sensor are shown in Figure 7.

However, not all sensors must contain sensitive components and conversion elements. If the sensitive element directly extracts electricity, it can also be used as a conversion element. In the actual production design, there are many sensors that combine sensitive components and conversion components. For example, when the measured temperature difference is detected, the thermocouple sensor directly exits the electromotive force. Piezoelectric vibrators, piezoelectric pressure sensors, photoelectric devices, etc. are all sensors. Since the output signal of the sensor is usually very weak and one or more interference signals are usually mixed, a signal conditioning circuit is needed to amplify, filter, and configure it. The type of measurement circuit is mainly based on the sensor detection principle, which depends on the type of conversion element, such as bridge circuit, high-impedance input circuit, and differential amplifier circuit. This article uses the row scan method to scan the flexible tactile sensor array. In order to accurately determine the position of the force, the row and column signals collected each time need to be consistent with the row and column signals of the actual sensor array. The scanning circuit of the flexible tactile sensor array uses a piece of four-bit binary counter to change from 0000 to 1111 in the process of cyclically collecting signals. When the counter is 0000 , the signals of the first row of the flexible tactile sensor array are collected, and when the 
TABLE 3: Familiar analog multiplexers.

\begin{tabular}{lccc}
\hline Category & Model & Name & Features \\
\hline Analog switch & CD4066 & Four-way analog switch & Four groups of independent switches, two-way \\
& transmission & \\
& CD4051 & 8 out of 1 analog switch & Level shift, two-way transmission, address selection \\
& CD4052 & Dual 4 out of 1 analog switch & Level shift, two-way transmission, address selection \\
Multichannel analog & CD4053 & Three-way, two-way analog switch & Level shift, two-way transmission, address selection \\
switch & CD4067 & Single 16-channel analog switch & Level shift, two-way transmission, address selection \\
& CD4097 & Dual 8-channel circuit analog switch & Level shift, two-way transmission, address selection \\
& CD4529 & Dual four-way or single eight-way analog & Level shift, two-way transmission, address selection
\end{tabular}

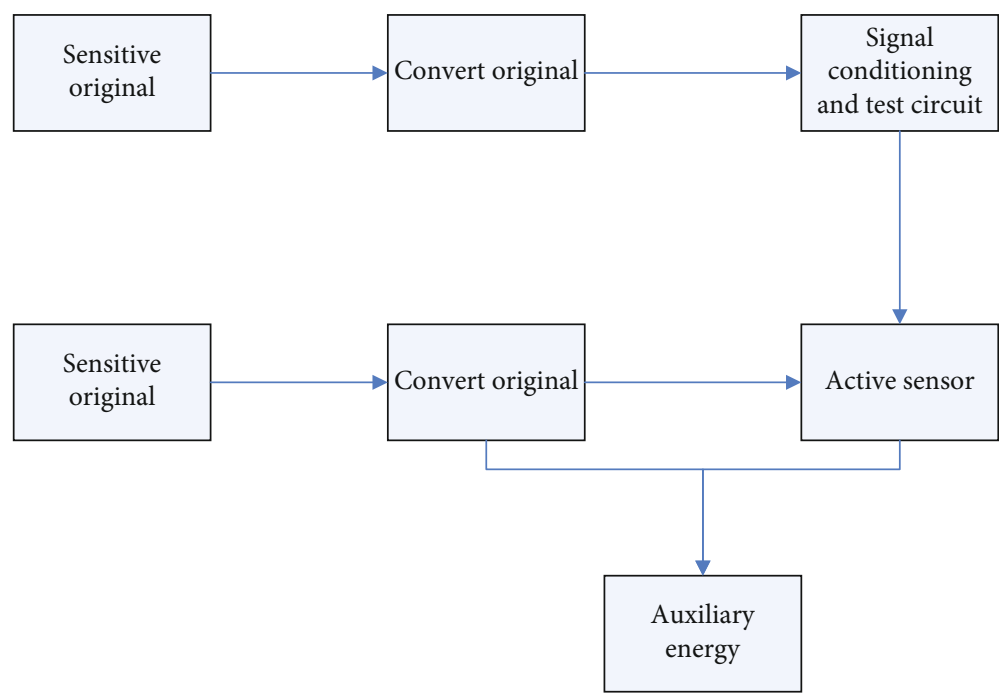

FIGURE 7: Diagram for sensor's composition.

counter is 0001, the signals of the second row of the flexible tactile sensor array are collected, and so on. Therefore, in this section, a digital signal acquisition program is designed to display the position of the scan line.

\section{Conclusions}

This article is aimed at developing a set of principle systems that can monitor walking energy consumption in real time. It mainly uses flexible array pressure sensors to detect plantar pressure distribution to obtain the user's gait information in real time and design it into the form of an insole, which is embedded in the sole. In the process of exercise, energy consumption is a very illusory concept after all. The user has a fuller and more specific understanding of speed, distance, and exercise time. Therefore, while effectively estimating exercise energy consumption, it must also be able to record the user's exercise. Time, estimate the speed and distance of the user. Based on these requirements, this article needs to establish two estimation models; one is the estimation model of walking speed, and then, the movement distance is calculated; the other is the estimation model of walking energy consumption, which can estimate the energy consumption of users in real time. These two models are the software part of the embedded sports energy monitoring principle system based on the flexible array pressure sensor insole. Theoretical analysis and experimental research show the new type of rubber-based touch sensor conductive plate and the information collection, analysis, and processing studied in this article. The system is completely feasible and can achieve accurate collection of large-area touch collection information. This is a good method and example, touch sensor panel. This development provides a new method and lays the foundation for the development of smart robotic clothing. The research prospects of this article are as follows. The experiments in this article are objective, the data are real, and the estimation model and experimental process established are reproducible and can stand the test, but they are only preliminary exploratory experiments. For the verification and affirmation of the entire experimental process, there are still many areas for improvement.

\section{Data Availability}

No data were used to support this study. 


\section{Disclosure}

We confirm that the content of the manuscript has not been published or submitted for publication elsewhere.

\section{Conflicts of Interest}

There are no potential conflicts of interests in our paper.

\section{Acknowledgments}

This work was supported by the Guangdong Philosophy and Social Science Planning Project under Grant No. GD20CTS02.

\section{References}

[1] D. Ning and L. Yijun, "Research on the reform of volleyball league in my country," Sports Culture Guide, vol. 20, no. 12, pp. 101-105, 2016.

[2] M. Zhou, X. Li, Y. Wang, S. Li, Y. Ding, and W. Nie, "6G multisource information fusion based indoor positioning via Gaussian kernel density estimation," IEEE Internet of Things Journal, 2020.

[3] Z. Lui, J. Li, A. Wang, J. Jia, and J. Wang, "UWB indoor positioning accuracy calibration method based on motion capture system," Journal of Surveying and Mapping Science and Technology, vol. 34, no. 2, pp. 147-151, 2017.

[4] Y. Wang, T. Lu, and H. Yonghua, "Distortion recovery of human motion capture data based on bone constraint," Computer Systems \& Applications, vol. 27, no. 5, pp. 19-27, 2018.

[5] Z. Liang, "The current situation of volleyball teaching in colleges and universities and the improvement of teaching mode," Journal of Heihe University, vol. 8, no. 10, pp. 120-121, 2017.

[6] Y. Liu, Y. Feng, Q. Li, Y. Wan, C. Lou, and J. Lou, "Research on characteristics of kinematic parameters of stilt method based on 3D motion capture technology," Journal of Beijing University of Traditional Chinese Medicine, vol. 41, no. 3, pp. 235241, 2018.

[7] F. Huang, Q. Zhang, H. Wang, L. Wang, C. Sun, and Y. He, "Application of motion capture technology in the study of biomechanics of massage techniques," Chinese Medicine Orthopedics, vol. 29, no. 11, pp. 41-43, 2017.

[8] H. Yizi, "Motion capture technology process and data processing_- facial motion capture and data processing," Modern Film Technology, vol. 3, no. 1, pp. 39-42, 2016.

[9] T. Jinghua and Y. Yiguo, "The application of optical threedimensional motion capture in the research of motion system," Hainan Medicine, vol. 29, no. 14, pp. 111-114, 2018.

[10] Y. Tingting and H. Bin, "Human gait analysis based on 3D motion capture technology," Digital Technology and Application, vol. 5, no. 2, pp. 232-233, 2017.

[11] C. Zimin, "Human motion segmentation and annotation technology based on motion capture data," Tomorrow Fashion, vol. 20, no. 13, pp. 299-299, 2017.

[12] T. Wu, X. Wu, D. Hu, Q. Wang, and F. Wang, "Research progress of shoulder joint kinematics analysis based on optical motion capture system," Chinese Electronic Journal of Shoulder and Elbow Surgery, vol. 4, no. 4, pp. 248-250, 2016.

[13] Y. Hou, X. Zhang, B. Li, and W. Li, "Application research of motion capture technology in the virtual environment of sub- station," Journal of System Simulation, vol. 28, pp. 2632-2637, 2016.

[14] C. Ningbo, W. Zhiming, and X. Cundong, "Analysis of human skin deformation during cycling based on motion capture," Journal of Textile Research, vol. 39, no. 9, pp. 120-126, 2018.

[15] G. Chunyu, "Motion mechanical parameters and related influencing factors of lumbar spine rotation manipulation in motion capture sitting position," China Orthopedics and Traumatology, vol. 32, no. 9, pp. 802-806, 2019.

[16] Z. Haifeng, Z. Can, L. Meixiao, S. Cuirong, and P. Yin, “Analyzing the movement ability of hip joints based on motion capture technology," Chinese Tissue Engineering Research, vol. 941, no. 12, pp. 17-21, 2021.

[17] H. Tianyu and L. Qi, "A review of research on motion capture technology and its application in sports," Electronic Measurement Technology, vol. 42, no. 3, pp. 146-152, 2019.

[18] Z. Chao, J. Longbin, and H. Cheng, "Cue-guided facial motion capture and data virtual reuse method," Computer Science, vol. 45, pp. 198-201, 2018.

[19] P. Shi, J. Lai, P. Lu, and S. Bao, "Real-time attitude measurement algorithm for multi-rotor aircraft based on motion capture system," Navigation and Control, vol. 17, no. 72, pp. 104-109, 2018.

[20] F. Liao, S. Jinlong, and G. Xiao, "Three-dimensional motion capture method of point-like feature flexible object," Computer Systems \& Applications, vol. 27, no. 7, pp. 230-235, 2018.

[21] J. Li, C. Gao, M. Feng et al., "Research on the related issues of motion capture, shaking, pulling and poking in the treatment of ankle sprain movement," Chinese Journal of Orthopaedics and Traumatology, vol. 26, no. 9, 2018.

[22] R. Wen, "Research on auxiliary training system based on Kinect motion capture technology," Electronic Design Engineering, vol. 27, no. 7, pp. 75-78, 2019.

[23] N. Jingde, "Application analysis of motion capture technology in film and television animation production," Theoretical Research and Practice of Innovation and Entrepreneurship, vol. 3, no. 5, pp. 149-150, 2020.

[24] X. Zhong, J. Li, J. Song, W. Lou, and W. Sun, “Application of motion capture technology in power safety simulation training," Information Technology, vol. 43, no. 7, pp. 170-174, 2019.

[25] L. Bing, "The application of motion capture technology in film and television animation production," Television Technology, vol. 42, no. 10, pp. 46-49, 2018.

[26] L. Shuhua, "Research on the application of performance motion capture technology in film and television performance animation," Television Technology, vol. 42, no. 10, 2018.

[27] W. Defang, "Research on the digital processing technology of the original data of the Anzhao dance of the Tu nationality based on the motion capture system," Journal of Science \& Technology Economics Guide, vol. 27, no. 28, pp. 39-39, 2019.

[28] W. Shixiang, "Research on digital protection of ethnic dances based on motion capture technology," Public Standardization, vol. 309, no. 16, pp. 153-154, 2019.

[29] S. Chen and M. Xiangxu, "Application of performance motion capture technology in film and television performance animation," Southern Agricultural Machinery, vol. 50, no. 4, p. 187, 2019.

[30] M. Zhou, Y. Wang, Y. Liu, and Z. Tian, "An informationtheoretic view of WLAN localization error bound in GPSdenied environment," IEEE Transactions on Vehicular Technology, vol. 68, no. 4, pp. 4089-4093, 2019. 
[31] S. Yao, L. Li, Y. Wang, F. Chang, and Y. Zeyuan, "Whole body motion capture method based on dual Kinect adaptive weighted data fusion," Journal of Chongqing University of Technology, vol. 33, no. 9, pp. 109-117, 2019.

[32] C. Wei, "Application of motion capture technology in 3D computer animation production," Automation and Instrumentation, vol. 32, no. 12, pp. 143-144, 2017. 